\title{
Conceptos, evidencia y realidad. El caso del "encierro" 1 del sitio arqueológico Nuevo Corintio (L-72NC), Guápiles, Limón
}

\author{
Manuel Castillo-Poveda
}

Invitado nacional

Universidad de Costa Rica

povedaarq@gmail.com

Recibido: 1 de Septiembre del 2016 Aprobado: 4 de Agosto del 2017

\begin{abstract}
Resumen:
El presente trabajo ahonda en la relación entre el lenguaje, la ciencia y la arquitectura, mediante el análisis y la deconstrucción de un término referido a un espacio arquitectónico precolombino denominado "encierro" en el sitio arqueológico Nuevo Corinto (L-72NC). Se evidenciará cómo esto repercute no solo en un cambio conceptual, sino también en el entendimiento de la realidad social llevada a cabo en este sitio por las sociedades antiguas.
\end{abstract}

Palabras clave: arqueología, arquitectura, deconstrucción, evidencia, lenguaje, realidad.

Concepts, evidence and reality. The case of "enclosure" 1 from the archeological site Nuevo Corinto (L-72NC), Guápiles, Limón

Abstract:

The present work delves into the relationship between language, science, and architecture, through the analysis and deconstruction of a term referring to a pre-Columbian architectural space called "encierro" in the archaeological site Nuevo Corinto (L-72NC). It will be explained how this implies not only a conceptual change, but also a change in the understanding of the social reality that happened in this space.

Keywords: archeology, architecture, deconstruction, evidence, language, reality.

\section{Manuel Castillo-Poveda}

Bachiller y licenciatura en Antropología con énfasis en arqueología de la Universidad de Costa Rica. Ha laborado en investigación, evaluación, rescate, promoción y restauración del patrimonio arqueológico costarricense, así como para instituciones como el Museo de Costa Rica, Universidad de Costa Rica, Ministerio de Cultura y de forma independiente. Entre sus líneas de investigación atiende a las temáticas referentes al desarrollo epistémico en las ciencias sociales, la destrucción del discurso científico y la constitución ontológica de la ciencia entre otros temas. 


\section{Introducción}

E objetivo del presente texto es ahondar en la práctica de nominar aspectos culturales y datos científicos que carecen de una sustentación adecuada; esto tomando como base la disciplina de la arqueología. Tal abordaje busca que cada término asignado a una expresión cultural de una población antigua tenga una fundamentación congruente con la evidencia; de esta manera, se podrán evitar errores, así como despejar contradicciones políticas, de historia o significado que tenga el concepto otorgado.

Las investigaciones referentes a los espacios denominados como "plazas" han sido de carácter exploratorio y descriptivo; acercamientos que no han vislumbrado el posible uso ni significado de dichos lugares (Castillo, 2014, p. 23).

Para desarrollar este objetivo, el presente artículo se sustenta en el trabajo denominado "Paisaje y arqueología: Arquitectura y conceptualización de las manifestaciones P-01, P-02, P-03 "encierro" 1 en el sitio arqueológico Nuevo Corinto (L-72NC), Caribe Central, Costa Rica" el cual se encuentra dentro del proyecto de investigación de la Universidad de Costa Rica "Nuevo Corinto: una aldea cacical" y en empresas cuyo objetivo fue el abordaje del sitio arqueológico Nuevo Corinto (L-72NC), y dar explicación a distintas manifestaciones culturales llevadas a cabo en él1.

El sitio ${ }^{2}$ arqueológico en donde se desarrollará el análisis conceptual posee 4 espacios arquitectónicos que, por tradición arqueológica, se han denominado de una misma manera ("plazas"). Dicha referencia no posee fundamento sustentado en evidencia precolombina; sin embargo, "se les adjudica una gran relevancia en las sociedades precolombinas tardías, esto debido a su presencia concomitante en los asentamientos, nuestra asociación cognitiva e histórica con tales vocablos y su aplicación a contextos antiguos" (Castillo, 2014, p. 22).

En cuanto a estos espacios precolombinos, en un principio fueron referidos como "plazas" (Salgado, Hoopes, Arias, Maloof, \& Aguilar, 2009); dicha denominación cambió a una nomenclatura referida como P-(numeración consecutiva). Cuatro años después, se nombró uno de ellos como "encierro" 1 Salgado, Hoopes, Aguilar \& Fernández, (2013). Este, específicamente, será abordado en el trabajo. La justificación de dicho cambio de nominación no es clara y por ello se plantea profundizar en el aspecto conceptual de cada uno de estos títulos y entender su significado etimológico e implicaciones sociales, así como corroborar su concordancia fáctica con las características arquitectónicas que presenta.

Dicho ejercicio permitirá reflexionar sobre la acción de registrar o nombrar algún aspecto de una sociedad antigua, subrayando la importancia del lenguaje como edificador de la realidad y medio de entendimiento de esta. El presente argumento será perceptible en el proceso de la deconstrucción de determinado concepto, ya que dentro de tal se

1 "Acción o efecto de manifestar" (Pelayo y Gross, 1972, p. 554), en donde "manifestar" se refiere a "dar a conocer" (Pelayo y Gross, 1972, p. 249), cosa que implica un "proceso psíquico, accesible directamente al hombre [sic] por su conciencia" (Brugger, 2005, p. 132), la cual infiere "un saber concomitante acerca de la existencia psíquica propia y de los estados en que en un momento dado esta se encuentra" (Brugger, 2005, p. 124).

2 "un sitio o una entidad, es definido y conceptualizado siempre a partir de algo, está siempre revelado como un lugar, no pudiendo tener previamente una conciencia de él como cualquier forma de no-lugar. Para él, por tanto, un paisaje es una red de lugares relacionados que han sido gradualmente revelados mediante las interacciones y actividades habituales con las personas, a través de la proximidad y la afinidad que éstas han desarrollado con ciertos emplazamientos y a través de acontecimientos importantes, festividades, calamidades, sorpresas y otros momentos que han llamado su atención, haciéndoles recordar o incorporar a la memoria colectiva (Thomas, 1993, p. 173). buscará establecer relaciones y correspondencia entre un término específico con la materialidad presente, registrada o fáctica. Con este panorama, se pretende plantear una inferencia que comprende la dinámica del lugar abarcado, extendiendola a un área mayor, así como a otras edificaciones.

\section{Contextualización}

El sitio arqueológico base para la presente investigación se encuentra en Costa Rica, en la Región Arqueológica Central; específicamente, en la provincia de Limón, cantón de Pococí y distrito de Guápiles; en la Sub-Región Arqueológica Caribe Central (Hoja cartográfica: Guápiles, Lambert Oeste-Este: 548,550, Sur-Norte: 244.000 , Latitud: $10^{\circ}$ 13' 18", Longitud: $\left.83^{\circ} 53^{\prime} 06^{\prime \prime}\right)$, y a una altitud de 220 m.s.n.m. Su extensión es de 40 hectáreas, delimitándose 10 hectáreas a la concentración de estructuras, como de material cultural perteneciente a la época precolombina.

El sitio Nuevo Corinto (L-72NC) está relacionado con la ruta conocida como "Línea Vieja", nominación que remite a 1871, cuando se llevó a cabo la construcción de la línea férrea que se trazó de San José a Limón (Peraldo y Rojas, 1998, p. 103). Al momento de su construcción, se fueron vislumbrando una serie de sitios arqueológicos, entre ellos, el citado en este trabajo, que fue registrado por la arqueóloga Doris Stone (1958) en su publicación "Introduction to the Archaeology of Costa Rica (reportado por la misma autora como la Unión Norte y Sur) (Stone, 1978, p. 18). 
Figura 1: Ubicación del sitio Nuevo Corinto (L-72NC), con base en la Hoja cartográfica Guápiles, Escala 1:50 000 del IGN. Su ubicación con relación al territorio nacional (esquina superior izquierda).
Figura 2. Planimetría del sitio arqueológico Nuevo Corinto (L-72NC). Tomado de Salgado et al. (2013, p. 13), modificado por Castillo (2014).

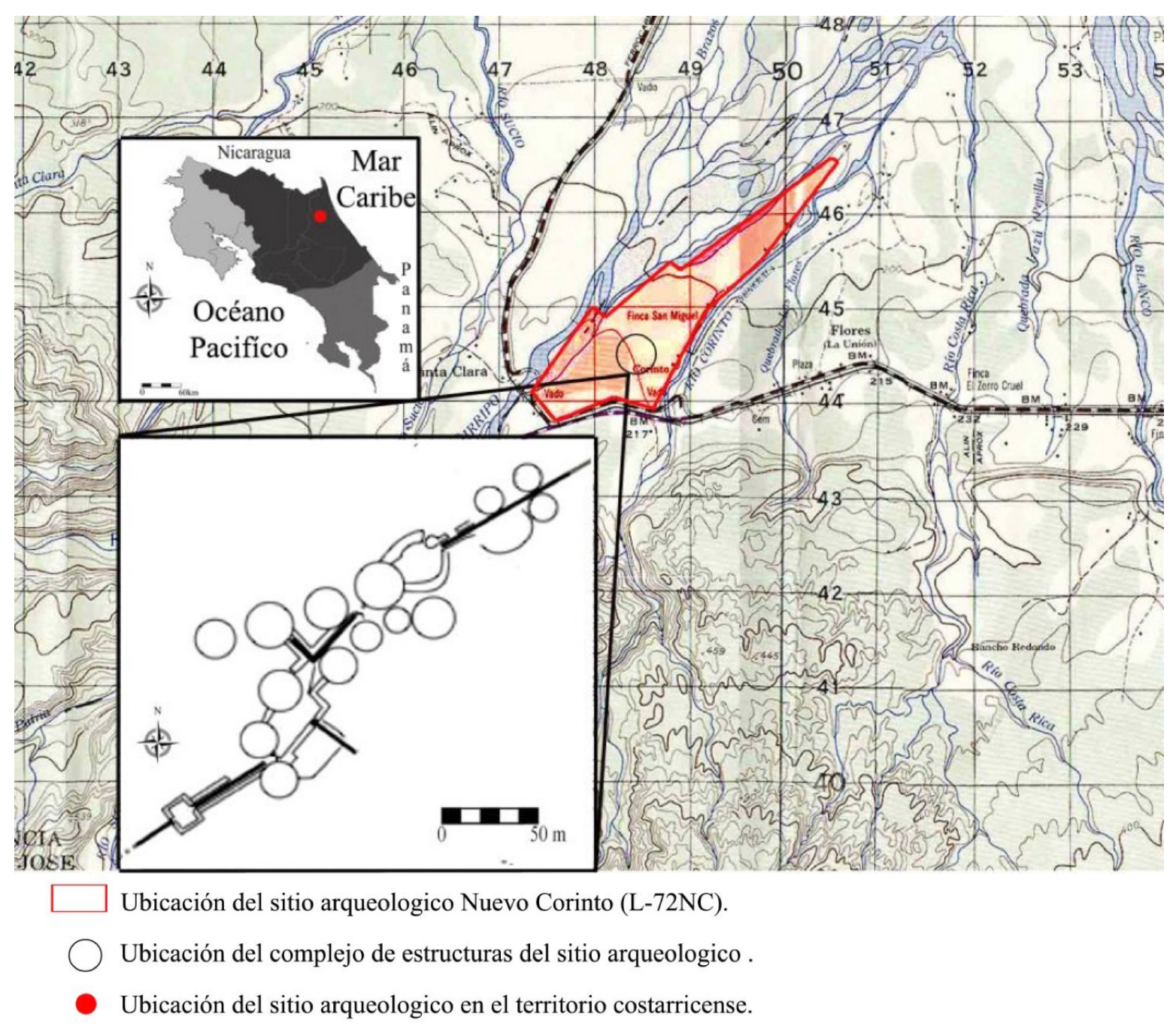

Aunque no se ha confirmado dicha ruta, el relieve e indicios de caminos que se infieren en los sitios arqueológicos relacionados, apunta a su posible existencia, que se emplazaría, aproximadamente, a $40 \mathrm{~km}$ de extensión en dirección Este-Oeste. En el caso de Nuevo Corinto, este se localizaría en el extremo Oeste de este trazado, ubicación que lo relaciona espacialmente con los sitios arqueológicos monumentales como lo son Las Flores (L-143LF) y La Manuda (L-130-LM), debido a la corta distancia que los separa.

Respecto al complejo de estructuras que comprende el sitio arqueológico, este goza de la presencia de 14 montículos, empedrados, dos calzadas, tumbas, muros, drenajes, así como cuatro diferentes espacios denominados como P-01, P-02, P-03 y "encierro" 1 , cada uno de los cuales presenta características diferentes. Aún así, se han denominado de una misma manera ("plazas"), acción que evoca la problemática de limitar el significado que estos lugares puedan evidenciar a una expresión generalizada y no a la relación de las cualidades que le conforman.

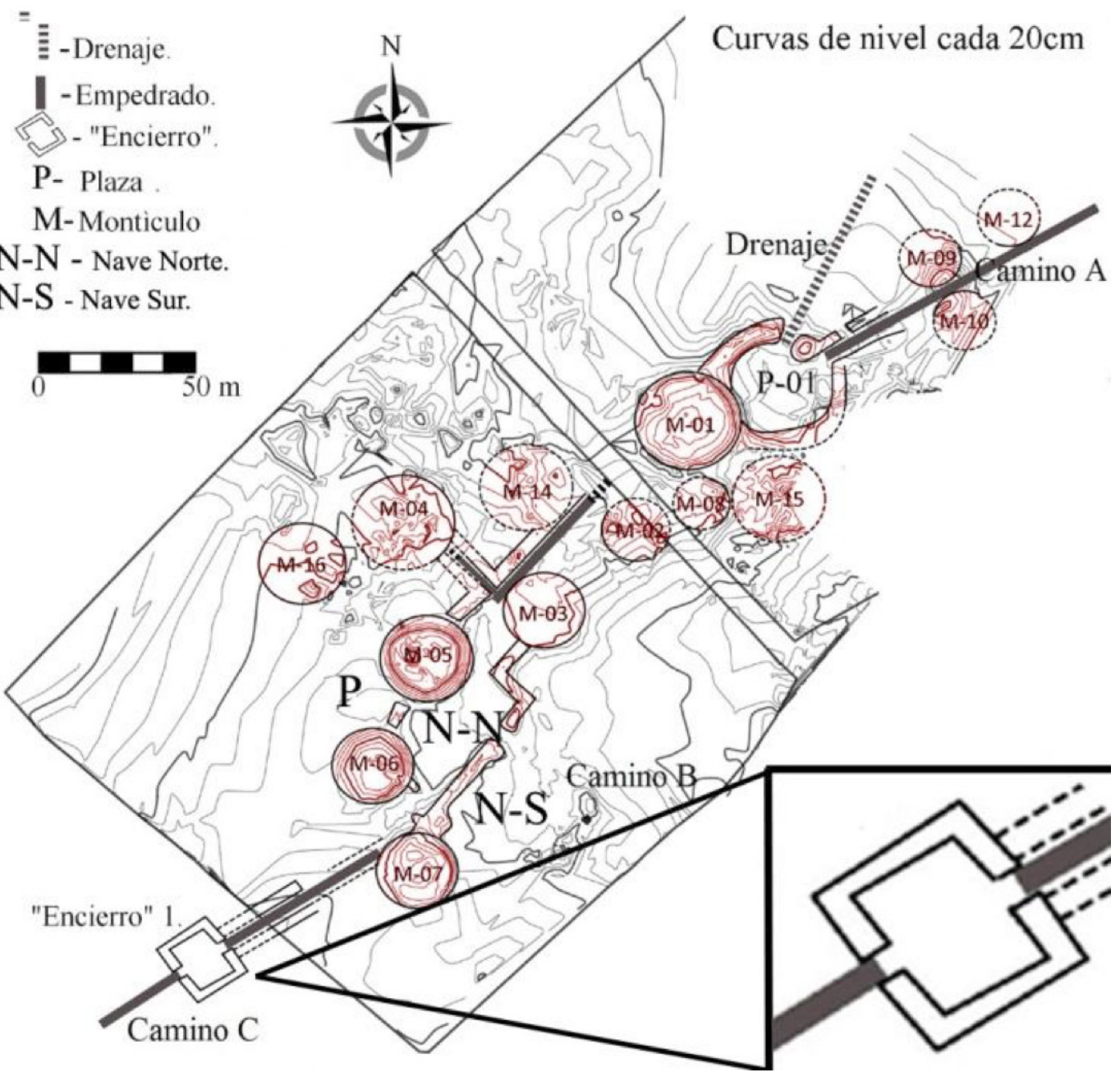


Figura 3. Ubicación de las excavaciones realizadas en el "encierro" 1 del sitio arqueológico Nuevo Corinto (L-72NC). Tomado de Salgado et al. (2013, p. 107), modificada por el autor.
Figura 4. Fotografía del fragmento de metate conformando parte del muro Sur del "encierro" 1 (Superior derecha); empedrado dentro del mismo espacio (superior derecha). Fuente: Autor
Las investigaciones realizadas en el sitio Nuevo Corinto -específicamente, las que abarcaron el espacio a abordar en este artículo- inician en el 2009, cuando se llevó a cabo una planimetría del complejo arquitectónico que contiene (Salgado et al., 2009); un año más tarde, se efectuó una limpieza de la estructura que le constituye, y, en el 2011, se ejecutaron diversas excavaciones, de las cuales se pudo obtener evidencia precolombina de tipo cerámica como lítica, además de registrar la disposición del empedrado perteneciente al "encierro" 1, como la configuración constructiva, en donde se visualizó la presencia de un metate fracturado como elemento cohesionador de uno de los muros que le conforman (Salgado et al., 2013).

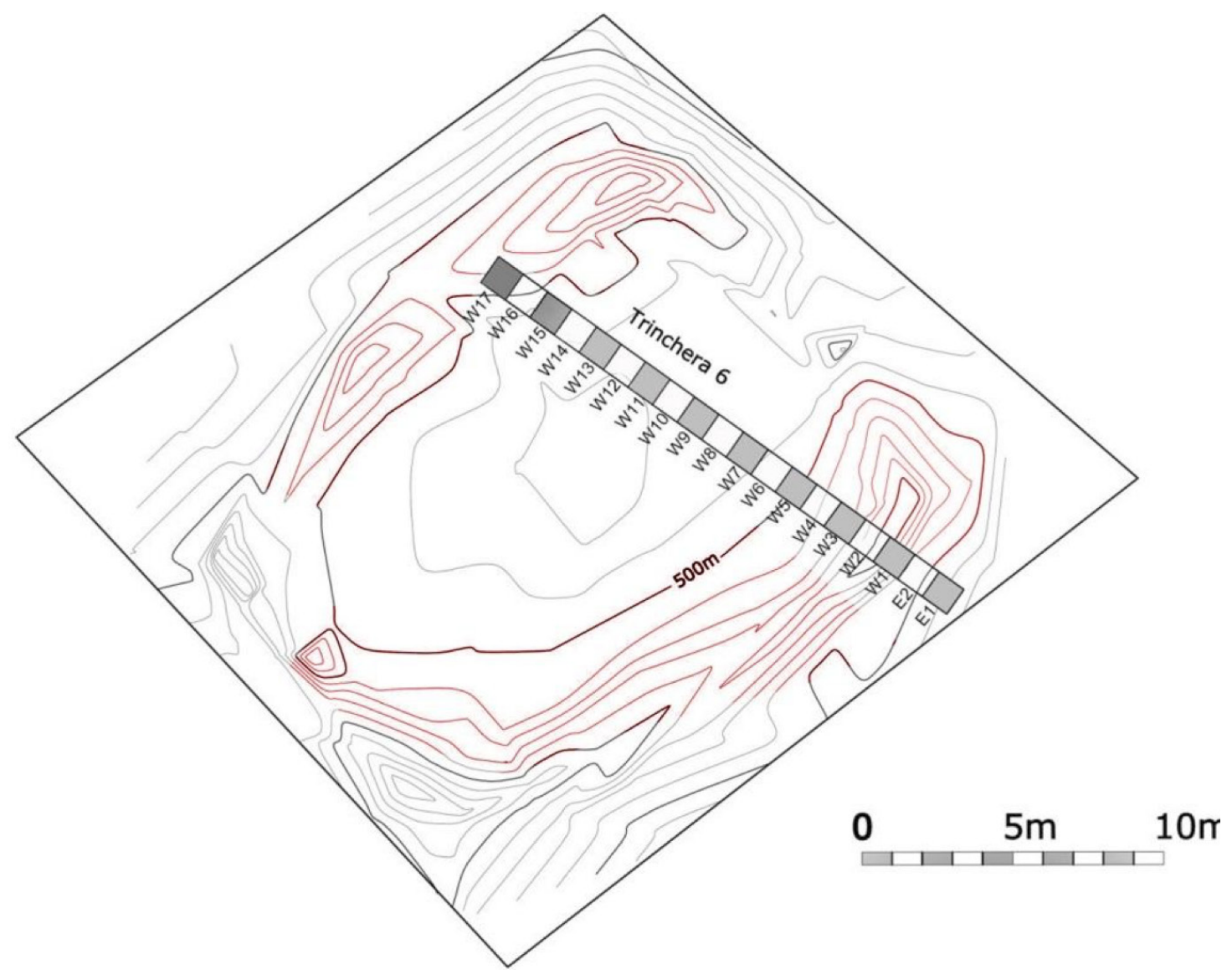

Este espacio referido como "encierro" es de forma cuadrangular, con un área de $357 \mathrm{~m}^{2}$, conformado por cuatro muros (con medidas de 21 metros y 17 metros de longitud y 2.5 metros de ancho), de los cuales dos tienen aberturas que se encuentran, aproximadamente, en la parte media de estos, y se presentan en las estructuras Noreste y en la Suroeste. Además, se registraron otros muros adyacentes a ambos lados de la abertura del muro Este, los cuales se extienden, aproximadamente, 5 metros a manera de líneas paralelas en dirección Este (Salgado et al., 2009, pp. 11, 13-19; Salgado et al., 2013, pp. 12, 19, 50-58, 70, 72, 73, 106-114).
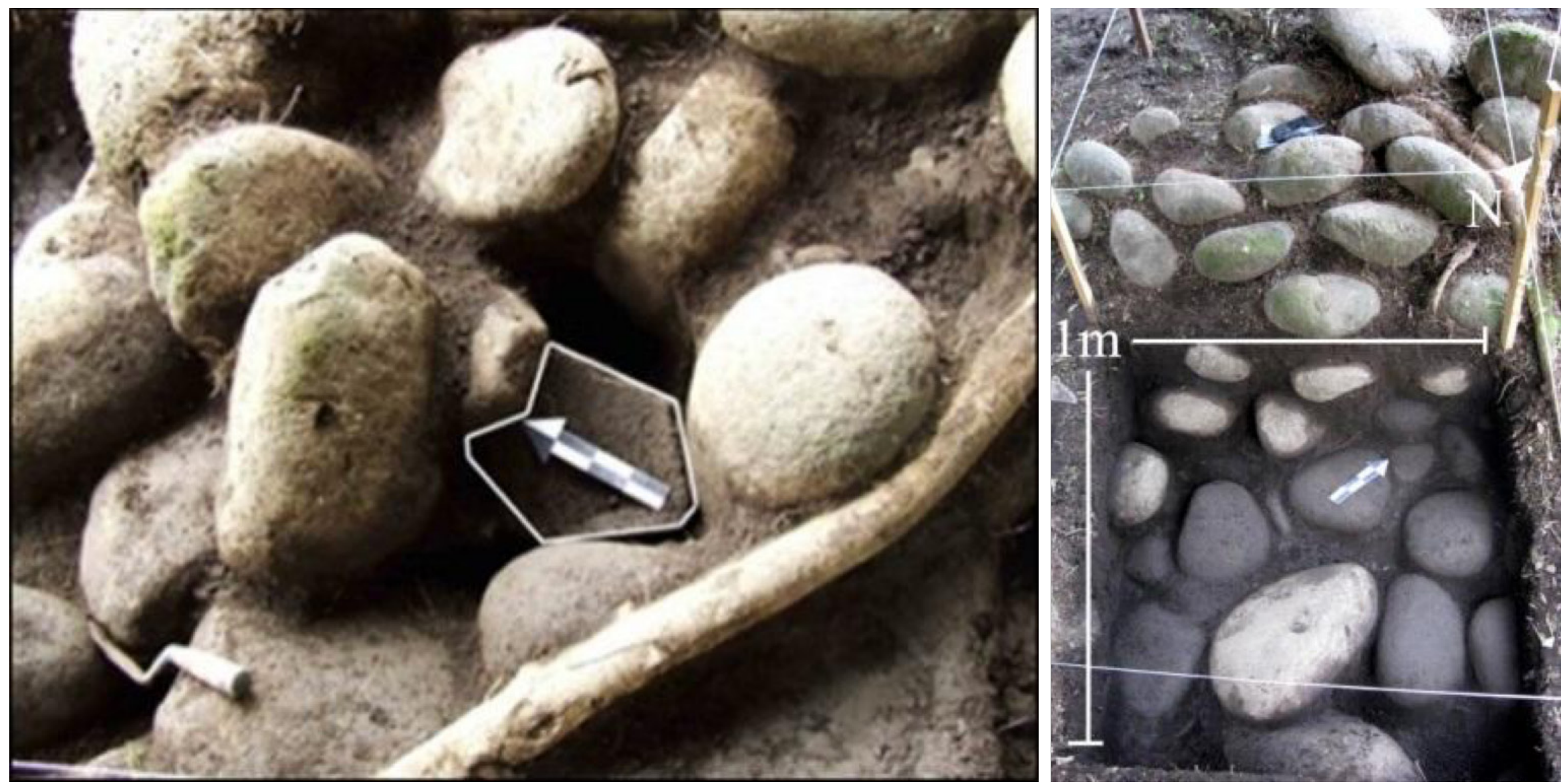

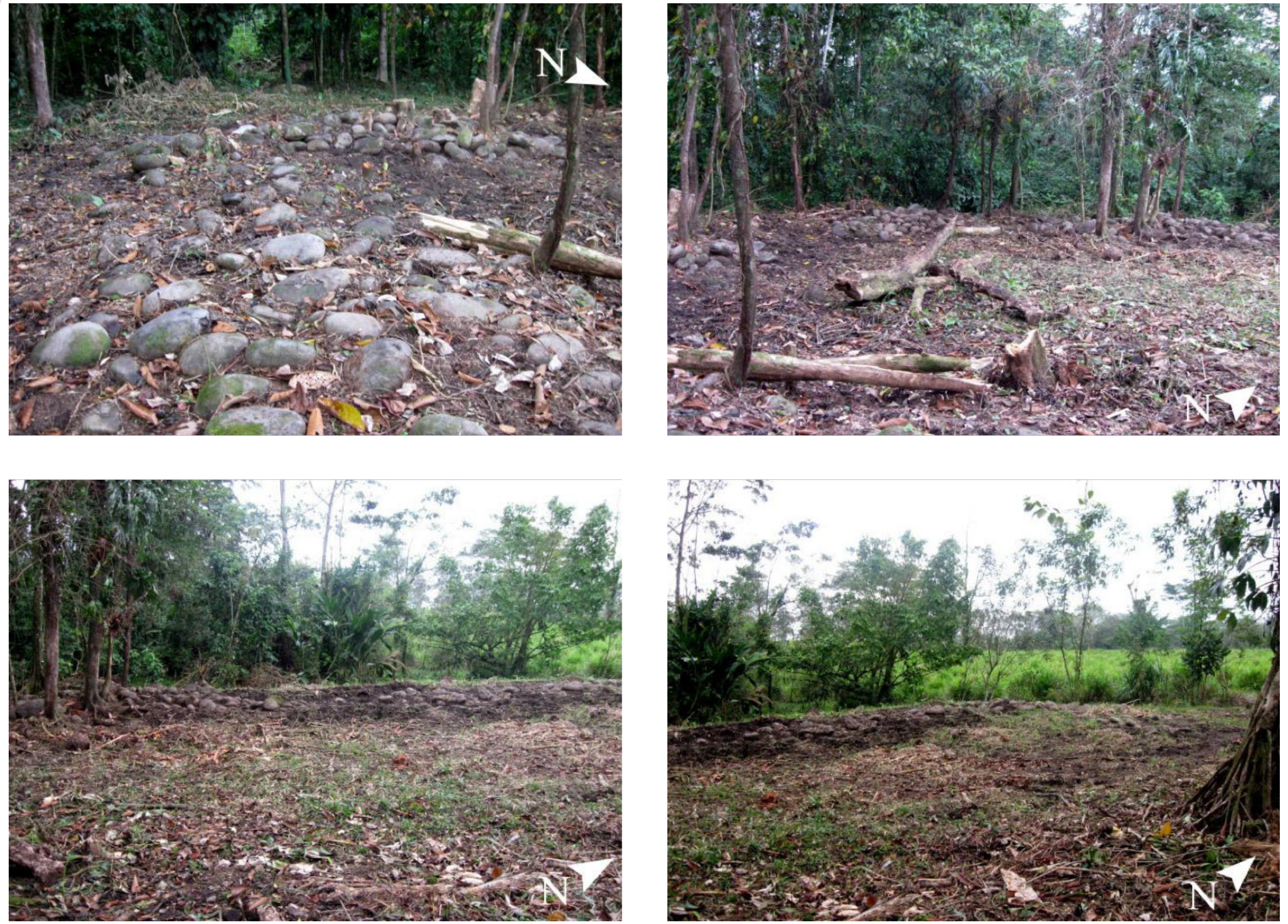

Figura 5. Fotografías de los distintos muros que $A$ conforman el "encierro" 1 del sitio arqueológico Nuevo Corinto (L-72NC). Tomado de Castillo (2014, p. 31).

\section{Justificación y problemática}

En el campo en las ciencias sociales es necesaria una constante revisión y una reflexión de lo investigado; de las bases históricas, arquitectónicas, políticas y filosóficas que sustentan la corriente de pensamiento que se acopla con este trabajo. Un elemento que el pensamiento y su difusión es el lenguaje, ya que es por medio de este que traducimos lo trabajado, además de dar a entender la realidad indagada.

Con el fin de poder captar una noción de los distintos matices sociales impresos en el lenguaje, Jacques Derrida (1930-2004) desarrolla el proceso de análisis denominado deconstrucción; mediante dicho abordaje se procura la búsqueda de un significado más cercano a lo que se quiso expresar y al contenido con que este fue emitido e impregnado: "La deconstrucción va a proporcionar los medios de interrelación en el campo de las oposiciones que critica y que es también un campo de fuerzas no discursivas" (Derrida, 1972, p. 21).

Con este tipo de aproximación, se busca el desarrollo intelectual del investigador, (al

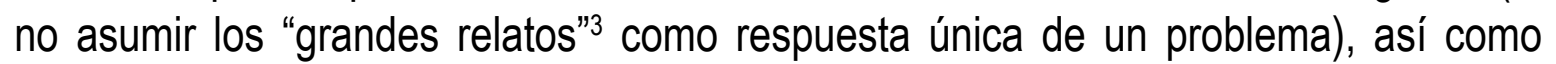
el esclarecer y exponer la carga histórica que contiene la realidad construida por su lenguaje:

(...) la premisa que todo sitio con complejidad arquitectónica corresponde a un cacicazgo y todo cacicazgo a un sitio tardío; esta secuencia de enunciados son asumidos como verdaderos por la tradición; así la estructura de asociación cognitiva empleada es a priori (Castillo, 2014, p. 115).

Dicha cuestión es de suma importancia debido al papel que juega el lenguaje en la construcción de realidades sociales, históricas y científicas, por lo que es necesario esclarecer cómo analizar la terminología utilizada en los discursos, con el objetivo de evitar contenido político erróneo que no se desea exponer o argumentos faltos de sustentación: "el pensamiento es la condición de posibilidad de que se dé el lenguaje; el concepto es causa del lenguaje en ese sentido" (Heidegger, 1927, p. 182).

El porqué de tal reacción es debido a cuestiones contextuales, ya que cada idea, fundamentaban las instituciones, como las prácticas públicas, sociales y políticas (Lyotard, 1994). 
$4 \quad$ Esto se sustenta en los trabajos de Florescano (1997); Gándara (1999); Gertz (1996); González (1996) y Litvak (1979).

$5 \quad$ Para abordar con mayor profundidad dicho tema, consultar Castillo (2014). palabra, escrito, enunciado científico u obra artística se construye y lee de acuerdo al medio socialmente construido en que se estudia ; sin embargo, el paso del tiempo provoca el descarte de unas acepciones, el fortalecimientos de otras y consolidación de pocas: "si se considera la historia como algo más que un depósito de anécdotas o cronología, puede producir una transformación decisiva de la imagen que tenemos actualmente de la ciencia" (Kuhn, 1962, p. 20). De ahí la constante revisión, discusión y argumentación de la tradición académica en la que se ubica este trabajo.

Todas estas acciones se llevaron a cabo bajo un presupuesto: no hay nada definitivo, todo está en construcción y cualquier dogma vigente en un marco histórico está presto a ser cambiado o, por lo menos, criticado, desfigurando la realidad que se ha querido imponer sin prestarse a su comprobación: "el progreso de una teoría de la racionalidad científica viene dado por descubrimientos de hechos novedosos, y por la reconstrucción en términos racionales de una porción creciente de historia teñida de valor" (Lakatos, 1978, p. 133).

Es por ello que personajes como Ludwig Wittgenstein (1889-1951) enfatizan en el lenguaje como un eje primordial para concebir la realidad. Estos argumentos son expuestos en su texto "Tractatus Logico-Philosophicus" (1921), y sostienen que la realidad en que vivimos puede ser sistematizada o referida con absoluta precisión, convirtiendo, de esta manera, en un plano inteligible por completo. Dicha perspectiva involucra varios problemas temporales, espaciales, culturales y de poder, que el mismo autor desarrollara en su segunda obra "Investigaciones filosóficas" (publicada en 1953).

Tal divergencia de aspectos evoca al problema ético relativo a la acción de decir qué es correcto 0 adecuado; esta condición conlleva una imprudencia existencial de cada ente referido: "para que una cierta proposición pueda afirmar un cierto hecho debe haber, cualquiera que sea el modo como el lenguaje esté construido, algo en común entre la estructura de la proposición y la estructura del hecho" (Wittgenstein, 1921, p. 2).

En el campo de la arqueología, el investigador posee un conjunto de cargas institucionales aunadas a la problemática mencionada; este profesional debe de entender a una población ajena a su persona por medio de un universo de aspectos (espacio, tiempo, lenguaje, ideología, entre otros), conjuntos de argumentos que validan su trabajo (método, teoría, política), así como la promoción de dichas investigaciones al público no especializado. Este panorama repercute en la comprensión de la historia, ahondando en el pasado de toda una población, su descendencia y sentando base para la construcción de una identidad 4 .

\section{Divergencia arquitectónico-conceptual}

Antes de iniciar con el desglose de cualidades que posee el "encierro" 1, cabe repasar y aclarar sus antiguas denominaciones; esto para tener una perspectiva de los distintos términos que se le ha asignado a un conjunto de evidencia y, por lo tanto, a las diferentes realidades construidas a través de este.

Este espacio, en primer lugar, fue llamado "plaza" (Salgado et al., 2009, p. 17); dicha investigación se centró en la planimetría de dicho lugar y su denominación respondió a la tradición académica de asociar dicho concepto a lugares amplios y sin ningún impedimento dentro de estos ${ }^{5}$. Este término fue cambiado al de "encierro" 1 (Salgado et al., 2013), sustentándose en sus características morfológicas, además de inferirse la posibilidad de encontrarse techado. De tal forma: "el rasgo antes conocido como Plaza 4 (P-4), ahora lo denominamos Encierro 1, pues sus características arquitectónicas y funcionales se asocian más bien con un espacio techado, destinado probablemente a un área de trabajo, debido a los materiales recuperados" (Salgado et al., 2013, p. 19).

Respecto al concepto de "encierro", en este se halla la acción asociada a la privación de la libertad, lo cual no coincide con las evidencias arquitectónicas como los empedrados extensos para el tránsito y las aberturas relacionadas a la estructura. En sí, habría una ineficiencia en la intención de cautiverio (de encerrar algo). La multiplicidad de manifestaciones culturales (material cerámico -decorado y no decorado- y lítico), tanto dentro, como fuera de este, no concuerda con la manifestación mencionada. Es por ello que se debe precisar la relación entre la evidencia y su interpretación, ya que podría esbozar un panorama erróneo del paisaje social del que fue parte este lugar. 


\section{Abordaje al concepto de "encierro" 1}

Como ejercicio y aplicación de un adecuado razonamiento sustentado en la evidencia que se posee, se ahondará en el análisis de las características arquitectónicas del lugar denominado "encierro" 1 del sitio arqueológico Nuevo Corinto. Esto debido a que dicho ámbito es eje de expresión de distintas facetas del ser humano, goza de gran relevancia, no solo con base en su funcionalidad, expresión artística, ingenieril e histórica, sino como elemento de discusión acerca de la relación del mismo con su entorno, sus percepciones, el entendimiento de los modos de vida y el contexto cultural en el que nos desenvolvemos, e incluso, en el que otros pudieron interactuar.

Debido a sus características (durabilidad ante el transcurrir del tiempo y el ser medio de expresión existencial de tendencias, ideologías, artísticas e ingenieriles), este tipo de manifestación cultural es un elemento capaz de reflejar acontecimientos, así como el pensamiento de personas y sociedades a través de distintas épocas; por lo tanto, evidencia para el entendimiento de una historia o contexto.

Esta expresión arquitectónica a trabajar es: "un espacio de forma cuadrangular, cuyas dimensiones remiten a $17 \mathrm{~m} \times 21 \mathrm{~m}$ y se encuentra parcialmente empedrado (aunque en mal estado de conservación)" (Castillo, 2014, p. 406). Otra característica que presenta es la de poseer dos aberturas (de 2 metros cada una), en los lados Noreste y Suroeste, de las cuales se desprenden caminos. Además, se localiza en un nivel topográfico mayor que al resto de estructuras de Nuevo Corinto, lo que potencializa la visibilización del sector Oeste del conjunto arquitectónico (P-05, M-05, M-06 y M-07).

Respecto a las aberturas, la ubicada al Noreste concuerda con el trazo de un camino que se dirige al resto de estructuras de Nuevo Corinto; destaca que entre "encierro" 1 y el resto de edificaciones del sitio, se registró la continuidad de un posible empedrado (detectado por medio de sondeo con barra metálica), relacionándolo espacialmente con el resto del complejo arquitectónico, infiriendo un sentido de direccionalidad con respecto a este lugar.

Se destaca que en dicho sector, en el año 2009, se aplicó la técnica geofísica denominada magnetometría, para la búsqueda de evidencia arqueológica, cuyos resultados fueron negativos (Salgado et al., 2009, p. 37). Por otra parte, en el 2010 se efectuó un sondeo sistemático con barra metálica que reveló la presencia de un empedrado disperso en dicha área (Salgado et al., 2013).

Cabe mencionar que dentro del "encierro" 1 existe una inclinación del terreno, la cual desemboca en el sector central, donde se encuentra un espacio longitudinal a modo de canal. Este funcionaría como guía en el tránsito por este lugar y de las aguas que podría captar. Además, destaca la presencia de un elemento lítico sobresaliente (por tamaño y color) fuera del espacio investigado en dirección a la abertura Suroeste, a manera de marcador.

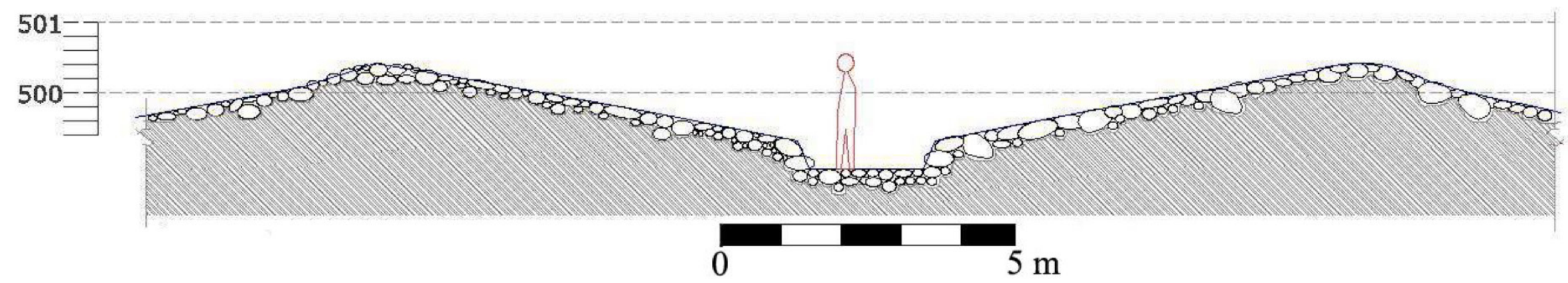

Figura 6: Grafico hipotético del perfil del "encierro" 1 del sitio arqueológico Nuevo Corinto (L-72NC). Tomado Salgado et al., (2013, p. 73).

Figura 7: Roca destacada por tamaño y color en dirección a la abertura Suroeste del "encierro" 1.

\section{A}

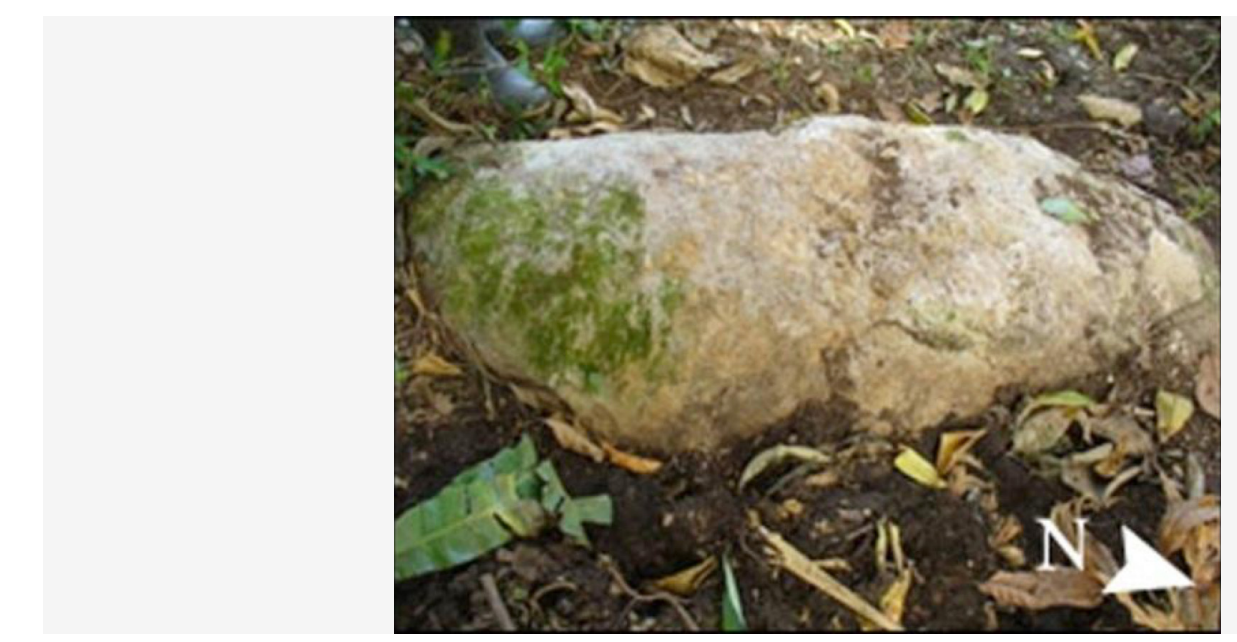


Figura 8: Gráfico de desplazamiento en el "encierro" 1 del sitio arqueológico Nuevo Corinto (L-72NC). Tomado de Castillo (2014, p. 350).
En cuanto al posicionamiento del espacio investigado, este presenta una noción de apertura. El transeúnte, al dirigirse hacia él y con orientación al resto de estructuras que comprende el sitio, pasa de un estadio de direccionamiento (la calzada) a un lugar más amplio ("encierro" 1), el cual controla el ingreso desde el Suroeste al resto del complejo arquitectónico.

Dicha noción se sustenta en la amplitud del espacio controlado por la arquitectura, la direccionalidad emitida por el cambio de nivel de suelo (evocado por el posible empedrado interno), la presencia de dos aberturas lineales delimitando el lugar referido, y el desplazamiento hacia este, por medio de la calzada, a un sector amplio culturalmente definido ("encierro" 1), ya que "el tránsito preludia un movimiento que es antes que nada, experiencia de espacio y construcción del tiempo, ya que tiene lugar en una extensión espacial y temporal concreta" (Criado, 1999, p. 32).

En la misma dirección, al llegar a la abertura Noreste de "encierro" 1, se posee una panorámica clara y abierta de la parte Oeste del conjunto arquitectónico; esto gracias a la diferencia topográfica y su posicionamiento (lo anterior podría resaltar el papel que jugaría la luz del sol en esta acción, ya que pareciera que no hay ningún techo de las edificaciones presentes en dicha dirección que impida su ingreso de manera directa). Posteriormente, su tránsito es de nuevo guiado, esta vez hacia P-02 o a la plaza (ver figura 2), mediante un camino empedrado (Camino C) con dicha dirección.

Es importante resaltar que dentro de "encierro" 1 existe una diferenciación de espacios, debido a la continuidad del camino hacia su abertura Noreste, marcada por una diferenciación de nivel (área central). Esto sugiere una división de áreas (los lugares adyacentes -sector Norte y Sur-), además de una relación de estas, tomando de eje el tránsito por él -el "encierro" 1-, donde el desplazamiento va en declive sobre el terreno, pasando de sólo una línea empedrada a estar rodeado por varias estructuras.

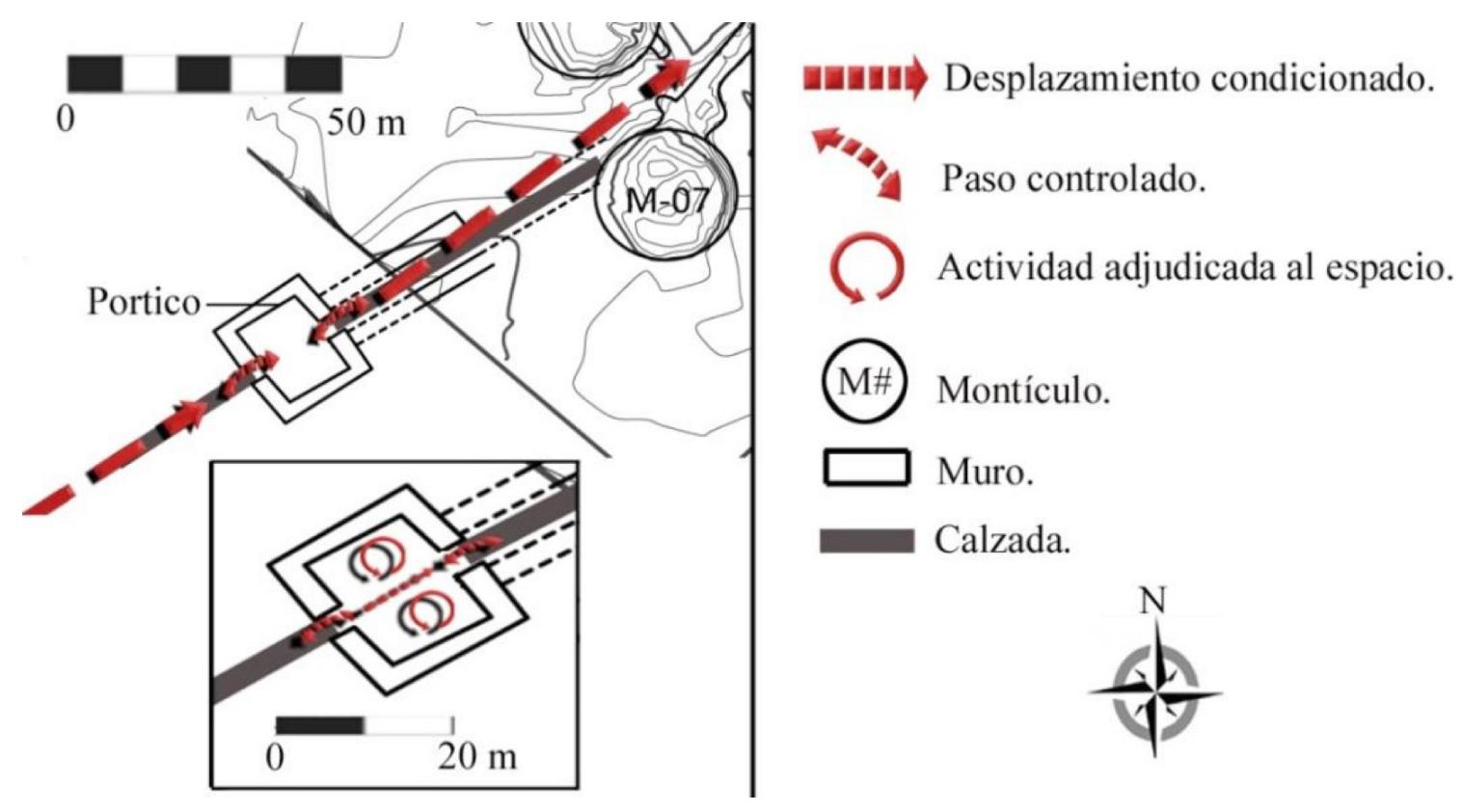

\section{Material arqueológico asociado al "encierro" 1}

En cuanto a las formas cerámicas presentes e inferidas según los fragmentos analizados, se registran ollas, escudillas, jarrones, además de platos. Estas se asocian a funciones como servir líquidos y alimentos, pero su carácter decorado infiere una exclusividad de uso, recalcando una diferenciación a caracteres cotidianos de las actividades involucradas.

También se destaca evidencia estructural referida a muros, aberturas, calzadas y paisajísticas, como lo es la utilización del desnivel como parte de la arquitectura presente (espacio longitudinal a manera de canal) (Salgado et al., 2013, p. 72). De tal forma, la presencia del empedrado permite inferir su no techado, basándose en el tamaño que deberían tener sus postes de apoyo para poder cubrir toda el área ( $21 \mathrm{x}$ $17 \mathrm{~m}$ ), además de la profundidad a la que estos tuvieron que ser enterrados para su estabilidad.

Respecto al material lítico, se presentaron lascas, núcleos, percutores, entre otros, que permiten inferir una zona de trabajo o taller dentro de "encierro" 1 (Salgado et al., 2014, pp. 230-234). 
Figura 9. Muestra de las reconstrucciones llevadas a cabo del material extraído del "encierro" 1 del sitio arqueológico Nuevo Corinto (L-72NC). Tomado de Castillo (2014, pp. 506-513).

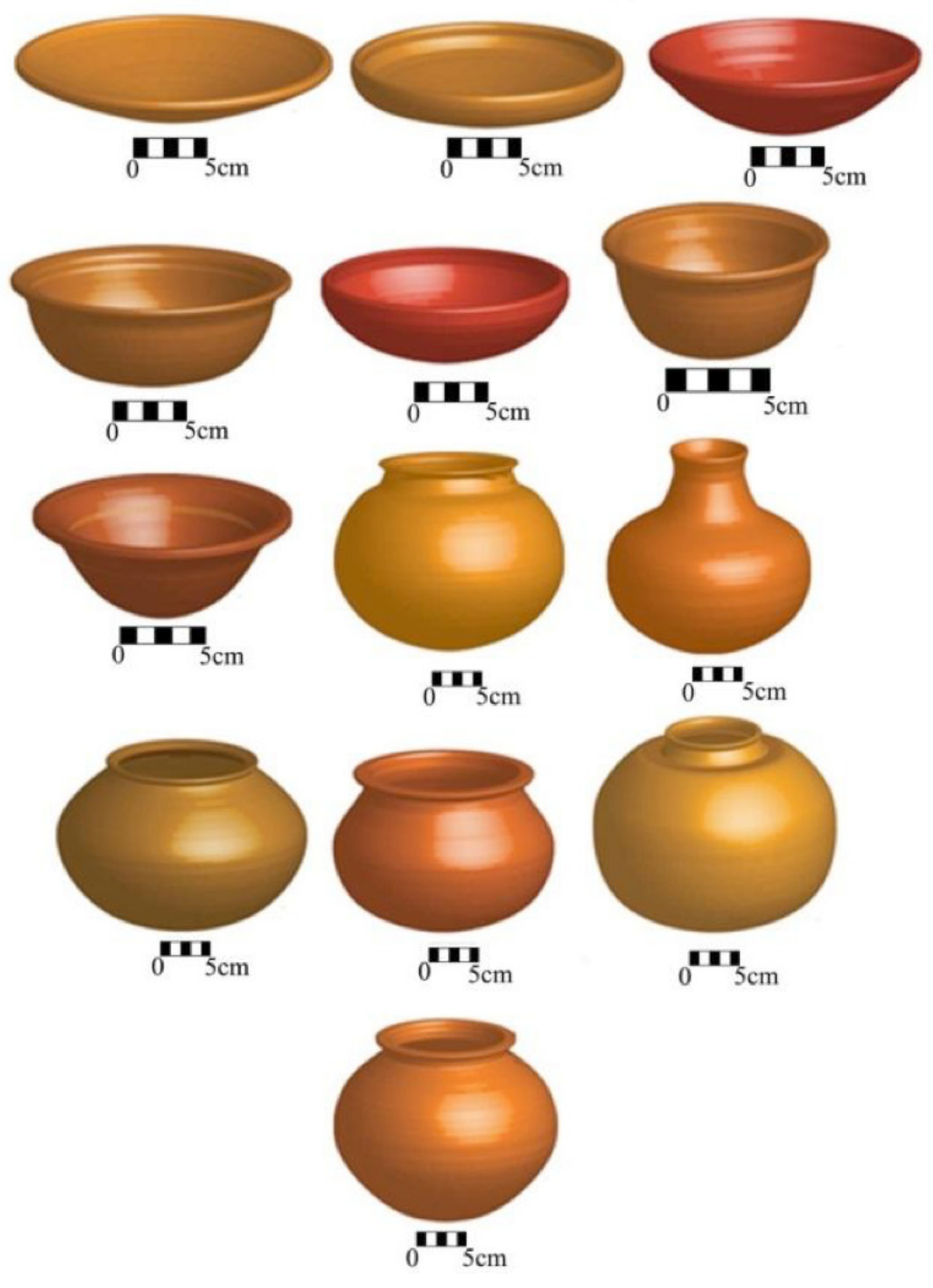

\section{Temporalidad del "encierro" 1}

Respecto a la datación relativa asignada a "encierro" ${ }^{6}$, se hallaron modos cerámicos asociados a la fase La Cabaña (850-1550 d.C.), el lapso entre La Selva-La Cabaña (500-1500 d.C) y La Selva (La Selva 300-850 d.C.). En cuanto a los tipos cerámicos identificados, estos se asociaron al primer lapso temporal citado.

A partir de esta evidencia, se considera que el clímax de uso de dicho espacio fue durante el lapso La Selva-La Cabaña. En dicho razonamiento se destaca el cambio o modificación de expresiones estilísticas sobre las funcionales (modos y tipos cerámicos), motivo por el cual predominan los tiestos asociados a La Cabaña (Salgado et al., 2013, p. 47).

\section{Abordaje conceptual}

Con base en la información proporcionada por la evidencia registrada en el "encierro" 1, la cual abarca distintas facetas del lugar investigado (morfológica, espacial, arquitectónica, temporal, funcional, paisajística, de tránsito y visual), a continuación se llevará a cabo un análisis de los conceptos adjudicados a este ("plaza" 4 y "encierro" 1):

\section{P-04}

La primer denominación que recibe este espacio es llevada a cabo por Salgado et al., (2009), donde se le denomina "plaza" o "P-04", y se le atribuye el ser la más pequeña que posee el sitio Nuevo Corinto (Salgado et al., 2014). El significado de dicho término refiere a un foro (forum) lugar de una ciudad donde se establecía el mercado, desde un punto de vista romano, a una "ágora [ayopá] "espacio abierto, centro del comercio[mercado], de la cultura y la política de la vida social" desde los griegos" (Mumford, 1979: 254, 272) y, desde una perspectiva arquitectónica, remite a un "lugar abierto o espacio público circundado por edificios" (Diccionario de arquitectura y construcción, 2013). Mientras que en arqueología se entiende como:

un espacio público específico dentro de un razonamiento de tránsito funcional en un conjunto de edificaciones y con un esquema de posicionamiento. Asimismo, se enfatiza dicho lugar como un sitio en el que se pueden llevar a cabo una variedad de actividades en las que se potencia la participación popular (Castillo, 2014, p. 360). 
De esta definición se extraen dos ideas esenciales para comprender el concepto de plaza en un plano material y social. La primera se refiere a una dinámica de tránsito, con lo cual evoca su negativa a encontrarse aislado de edificaciones, ya que necesita de estas para constituirse; la segunda es llevada a cabo por la población que transita continuamente de una manera no controlada por este conjunto de espacios y estructuras, en donde ejecutan determinadas prácticas en un área específica; esto por distintas cualidades de las manifestaciones culturales (recurrencia, ubicación, panorama, temperatura entre otros), que provocan su presencia reiterada en el mismo sitio.

La idea expresada introduce el tema de las prácticas cotidianas y la definición social por medio de su recurrencia. Lo cual se ve reflejado en características como la ausencia de una delimitación por estructuras propias y de edificaciones que se relacionan con la misma espacialmente, así como la presencia de evidencia material que difiere de un carácter singular.

En sí, la plaza se refiere a un lugar elaborado por la ejecución de prácticas cotidianas dentro de un conjunto de edificaciones, las cuales no delimitan este espacio, si no que le constituyen.

Este término contrasta con las características que proporciona la evidencia de los trabajos realizados en el espacio investigado ("plaza" 4), lugar que se encuentra delimitado por muros, controla el acceso y es direccionado por medio de aberturas, empedrados y su desnivel interno (drenaje [Castillo, 2014, p. 32; Salgado et al., 2013, p. 70]). Cabe destacar la integración de este espacio a una dinámica del complejo de edificaciones que posee el sitio Nuevo Corinto, la cual es señalada por evidencia como el camino C, el empedrado Noreste y la visibilidad direccionada hacia estos.

Sin embargo, la disposición de los muros delimitando un área de manera vertical y horizontal, al igual que el empedrado interno del espacio investigado (inclinado y con una zona a manera de canal en su parte media), implican una dificultad en su tránsito (Castillo, 2014, pp. 70, 409), lo que a su vez apunta hacia un desplazamiento unidireccional y controlado, cualidades que no concuerdan con el término asignado ("plaza").

\section{"Encierro" 1}

Respecto al concepto de encierro, la Real Académica de la Lengua Española se refiere a dicho término como la "acción y efecto de encerrar o encerrarse" o "lugar donde se encierra", ambas aseveraciones ligadas al verbo encerrar, el cual significa "meter a una persona o a un animal en lugar del que no pueda salir" (Real Academia Española, 2014), acción que contiene una negación de la voluntad del ser humano, ya que este no goza de la libertad de decisión durante la estadía en este espacio.

Esta noción de pérdida de la libertad debería estar materializada en un espacio delimitado mediante estructuras que dificulten su salida, ya sea por su altura o por aditamentos que evoquen peligro al ser traspasados. El motivo de encierro también se asocia a una actividad no aceptada, recalcada por la acción de no dejar salir. Esta actividad resulta en la restricción de cualquier tipo de dinámica externa, cortando el desarrollo de esta y siendo la respuesta ante un comportamiento o proceder impropio al grupo social en el que se encuentra.

Otra inferencia que se lleva a cabo con base en dicho término es su posicionamiento ante un complejo espacial, del cual el encierro forma parte; este se localizaría donde no impida la dinámica de desplazamiento del paisaje en que se encuentra, sino en relación con este o distanciado, con el fin hacer partícipe o exentar su visualización.

Retómese el trabajo realizado por Salgado et al. (2013), en donde llaman al espacio estudiado como "encierro" 1: se sustenta tal denominación en que "sus características arquitectónicas y funcionales se asocian más bien con un espacio techado, destinado probablemente a un área de trabajo, debido a los materiales recuperados" (Salgado et al., 2013). En este texto, y en concordancia con el trabajo de Castillo (2014), se difiere de dicha aseveración, no solamente por la ausencia de un techado.

De tal forma, la presencia del empedrado permite inferir su no techado. Esto con base al tamaño que deberían tener sus postes (de apoyo) para poder cubrir toda el área, así 
como la profundidad a que estos tuvieron que ser enterrados para su estabilidad. Vale decir que no se ha recuperado evidencia alguna que sustente la existencia de postes (o huellas de estos) en este lugar" (Castillo, 2014, p. 408).

Además, asegurar la existencia de este tipo de evidencia (techado) implicaría una dificultad para la acción de drenado (efectuada por el espacio referido como canal): "Si bien el drenado de aguas se lleva a cabo por medio de la inclinación del terreno a un punto fuera del lugar comentado; si hubiese un techo aquí habría menos evaporación y se anegaría más el terreno que comprende el espacio" (Castillo, 2014, p. 408). Por último, la presencia de techado no implica una funcionalidad específica "la presencia de un techo puede ser asociada a cualquier otro lugar 'funcional" (Castillo, 2014, p. 408).

Otra argumento esgrimido para justificar tal denominación es el de sus características arquitectónicas; según la definición de encierro, este debe ubicarse en un lugar donde la salida es difícil o solamente es obtenida si un sujeto fuera de este espacio la concede; es decir, debe significar un impedimento físico al ocupante (muros de gran altura y reducidos accesos).

Cabe señalar el empedrado no supone una relación con el término adjudicado (encierro), sino que responde a la dinámica de tránsito del complejo arquitectónico de Nuevo Corinto. Esto se deduce por la coherencia arquitectónica de empedrados relacionados a su abertura y un elemento lítico de características particulares (color y tamaño) que concuerda con el trazado de dichos caminos atravesando el "encierro" y direccionado al conjunto de estructuras (Castillo, 2014, p. 407). Se observan "aberturas y calzadas ligadas que provocaron un direccionamiento particular; ello por medio de un desnivel como parte de la arquitectura presente (drenaje)" (Castillo, 2014, p. 407).

Respecto a su función, se le adjudica ser un área de trabajo, aseveración que puede ser referida a cualquier otro espacio en un sitio arqueológico, ya que el verbo trabajar se liga a un sinfín de actividades, incluso algunas que no dejarían evidencia material; sin embargo, el estar encerrado significa imposibilidad de acción, por lo cual es difícil una actividad en este espacio que no sea la de esperar que se le permita salir, o la subsistencia alimentaria. En el caso que se produjera algún artefacto, este gozaría de un aspecto expedito, debido a las condiciones de su elaboración (encerrado, vigilado, sin acceso a herramientas ni condiciones aptas para dicha acción [luz, calor, materia prima]); aspectos que no concuerdan con la lítica hallada en el "encierro" 1: "Respecto al material lítico, se presentaron lascas, núcleos, percutores, escultórica, entre otros; lo que permite inferir una zona de trabajo o taller dentro de "encierro" 1" (Salgado et al., 2013, pp. 230-234), ni con la cerámica:

En cuanto a las formas cerámicas presentes tenemos ollas, escudillas, jarrones y platos. Estas se asocian a funciones como servir líquidos y alimentos, pero su carácter decorado infiere una exclusividad a quien se le da importancia de las actividades involucradas (Castillo, 2014, p. 416).

Por ello, el concepto de "encierro" 1 debe descartarse luego de su aplicación, ya que no concuerda con el conjunto de evidencia que proporciona este espacio. De aplicarse, llevaría a una concepción espacial y arquitectónica errónea, así como de las dinámicas sociales que aquí tenían lugar.

\section{Propuesta}

Debido a que ambas propuestas para denominar este espacio no concuerdan con el conjunto de datos que se ha logrado obtener en las distintas intervenciones en este lugar, se llevará a cabo el ejercicio de buscar un concepto que comprenda de forma adecuada la evidencia.

Para llevar a cabo dicho propósito se expondrá un recuento de las características que posee el espacio estudiado; cabe destacar que se implementarán dos premisas para la propuesta conceptual: la primera es que, para un entendimiento claro, la evidencia arqueológica deberá traducirse 0 asimilarse con base a una terminología moderna, ya que en esta tradición se formó el investigador. Cabe señalar que no se puede utilizar términos asociados a poblaciones antiguas, ya que hasta el momento no existe evidencia alguna que pueda ser adjudicada a estas. 
En segundo lugar, con base en la evidencia que ha proporcionado el conjunto de estructuras del sitio arqueológico Nuevo Corinto, se intuye un conjunto coherente y relacionado -física y paisajísticamente- en todo el espacio que comprende; por ello, la organización como desarrollo urbanístico es un tópico presente en la disposición de todas las estructuras de esta área.

Aunque existe una divergencia en la temporalidad relativa inferida del análisis del material arqueológico, se identifica una noción de desarrollo urbano, la cual ubica la edificación de estructuras en espacios específicos, creados por la reiteración de su uso. Por lo tanto, la temporalidad de edificación de estructuras en un espacio no necesariamente determina la función que ahí se llevaba a cabo; esta es presupuesta con lo que ahí se observa.

Reiterando las características que posee el "encierro" 1 del sitio arqueológico Nuevo Corinto, este es un lugar amplio, delimitado por dos muros en forma de dos paréntesis cuadrados, enfrente uno del otro, los cuales forman dos espacios a modo de aberturas, a Suroeste (en esta dirección se halla una roca externa al espacio investigado como marcador de camino) y Noreste, ligados a empedrados. Al trazo del primero corresponde al sitio El Abuelo ( $L-127 \mathrm{Ab}$ ), mientras que el segundo al conjunto de estructuras de Nuevo Corinto.

En la visibilidad hay una reducción al dirigirse a su abertura Suroeste. Aunque permite captar toda su área, al pasar la abertura Noreste se da una apertura mayor, observando el complejo de estructuras del sitio, entre ellas el montículo 5, 6, 7, la plaza y los enterramientos en el sector Noroeste (ver figura 2).

En cuanto al tránsito, este es guiado por medio de empedrados que se desprenden de las aberturas que posee este espacio, constriñendo debido a su reducida dimensión. Dentro del lugar analizado, el tránsito es de igual manera direccionado mediante el cambio en la altura del empedrado que concuerda con el trazo de las aberturas al resto del área interna.

Después de haber consultado diccionarios especializados en arquitectura ${ }^{7}$, puesto que este tópico es esencial para nombrar el sitio (modificación artificial de un espacio mediante la edificación de estructuras), se llegó al término "pórtico", el cual significa "espacio que antecede a un edificio o grupo de edificios" (Orosco, 2002, p. 140).

Tal elección es debido a la funcionalidad y disposición espacial del sitio respecto al resto de estructuras de Nuevo Corinto. La colocación de este pórtico, en sector Suroeste del complejo arquitectónico, diferencia paisajísticamente ámbitos delimitados, direcciona en relación con otra estructuras e implica una dinámica de desplazamiento (Castillo, 2014).

Vale mencionar que este cambio de paisaje sugiere al individuo el paso de una estadía natural, en la cual su desplazamiento es libre, así como su campo visual y su contacto con el suelo y vegetación, a uno donde el espacio es artificial; el transitar le es diferenciado por empedrados, hay constricción de movilidad debido al ancho de estos

$7 \quad$ Cabe destacar la dificultad de definición de estos espacios en la arquitectura, ya que estos se refieren tanto a partes de edificaciones, como a técnicas de estas. En este trabajo se llevó la revisión de los siguientes diccionarios especializados: Wolfgang (1964), Ward (1972) y Atrium (1998), entre otros. La lista de bibliografía correspondiente se encuentra en el trabajo de Castillo, 2014.

$8 \quad$ Para mayor información acerca de los espacios del sitio arqueológico Nuevo Corinto, refiérase a Castillo (2015). y a las aberturas del pórtico; la visibilidad es igualmente condicionada de una manera cuasi lineal a causa de los muros.

Aunque se da esta noción de control y direccionamiento, al traspasar el pórtico se lleva a cabo una apertura tanto visual como física, enfatizando el resto de estructuras, lo cual lleva a otro cambio de paisaje; este, aunque posee empedrados, caminos y espacios definidos, presenta un dominio visual de la arquitectura, lo cual, aunado a la presencia de la luz, a la edificación de chozas sobre montículos y a otras personas, produce asombro.

Son estas características las que llevan a la elección del término "pórtico", el cual es un espacio inserto en un componente de estructuras que antecede a un estado, donde la arquitectura domina todo a su alrededor; es decir, es un lugar que comparte una relación con el ambiente natural (espacio abierto y un contexto sin un direccionamiento) y con el artificial (direccionado, edificado físicamente, con un propósito y dinámica):

Con base en dichas características, "encierro" 1 es asociado a un espacio de control de acceso, más referido técnicamente a un "pórtico". Dicha definición se debe a que él aduce la unión de (o apertura a) un espacio diferenciado con el otro. Esto no sólo 
produce un cambio físico (arquitectónico) sino, también; de estado con respecto al transitar los mismos (Castillo, 2014, p. 409).

Este concepto no solo es coherente con la evidencia, sino que también se vincula con las estructuras que se encuentran a su alrededor, así como las que constituyen el complejo arquitectónico de Nuevo Corinto en una dinámica de desplazamiento, control, reunión y expresión de distintas manifestaciones culturales:

Referente al vínculo entre los distintos espacios investigados (vestíbulo, nave Norte, nave Sur, pórtico y plaza), estos muestran una continuidad con respecto a un posible motivo de conglomeración; así como a distintas funciones específicas ligadas a ellos (control [pórtico], reunión [plaza], suscitación [nave Norte]). En donde se ve reflejada la afirmación de su participación (pórtico), la expresión de su estancia (nave Norte y plaza) y una de las concreciones de su propósito (nave Sur) (Castillo, 2014, p. 445) (Ver figura 2). ${ }^{8}$

La presente investigación contrasta con trabajos anteriores en el área de edificaciones precolombinas puesto que se da explicación de lo referido y de por qué se da determinada denominación y, además, se da una descripción paisajística del sitio y de las posibles dinámicas que en él se llevaban a cabo:

Este espacio se asocia a una noción de apertura y control. En donde el/la transeúnte al dirigirse hacia el pórtico, y con dirección al resto de estructuras que comprenden el sitio, pasa de un estadio de direccionamiento lineal (la calzada) a un lugar más amplio (pórtico); el cual -a la vez-controla el ingreso desde el Suroeste al resto del complejo arquitectónico de Nuevo Corinto.

Dicho carácter se relaciona a la direccionalidad emitida por el cambio de nivel de suelo, evocado por el posible empedrado interno y la presencia de 2 aberturas lineales que delimitan el lugar referido, como el desplazamiento hacia éste, por medio de la calzada, a un sector amplio y culturalmente definido (pórtico).

En la misma dirección, al llegar a la abertura Noreste del pórtico se posee una panorámica clara y abierta de la parte Oeste del conjunto arquitectónico; esto gracias a la diferencia topográfica y su posicionamiento. Lo anterior podría resaltar el papel que jugaría la luz del sol en esta acción, ya que pareciera que ningún techo (en dicha direccionalidad) impediría su entrada. Aun así su tránsito es de nuevo guiado, esta vez hacia la nave Norte mediante un camino empedrado (Camino C) con dicha dirección. Fuera del pórtico el desplazamiento va en declive sobre el terreno, pasando de sólo una línea empedrada a estar rodeado por varias estructuras (Castillo, 2014, pp. 442443).

A lo largo de este texto se planteó una reflexión y una deconstrucción, no solamente a manera conceptual y fáctica, de un espacio precolombino, sino que se ahondó en la tradición que sustenta y determina las aproximaciones a una realidad ajena a nuestro contexto, y en las posibles implicaciones de esta labor interpretativa

Dicha acción es de suma importancia, ya que se desfragmenta una realidad ajena para edificar un discurso de carácter científico e histórico, aspectos que, como se mencionó en anterioridad, influyen en la constitución identitaria "La Historia sabida operaria, sostiene, como una pantalla que nos sustrae al suceder pretérito original y nos roba el mundo posible de la hora actual" (Heidegger, 1927, p. 220).

Es por ello que dicha construcción de la realidad debe, primero, sustentarse en evidencias y, segundo, evitar la influencia de vicios tradicionales o históricos, tales como adjudicar, catalogar e inferir determinadas cualidades, conceptos o actividades dados por la tradición. 


\section{Referencias bibliográficas}

Atrium. (1998). Biblioteca Atrium de La arquitectura actual. Barcelona: Atrium.

Brugger, W. (2005). Diccionario de filosofía. Barcelona: Herder.

Castillo, M. (2014). "Paisaje y arqueología: Arquitectura y conceptualización de las manifestaciones P-01, P-02, P-03 y "encierro" 1 en el sitio arqueológico Nuevo Corinto (L-72NC), Caribe Central, Costa Rica". Tesis para optar por el grado de Licenciatura en Antropología con énfasis en Arqueología. Facultad de Ciencias Sociales, Escuela de Antropología. Universidad de Costa Rica, San José.

Castillo, M. (2015). Conceptualización, caracterización y deconstrucción del término Nave en la arqueología: un acercamiento a los espacios P-02 y P-03 del sitio arqueológico Nuevo Corinto (L-72NC). RevistArquis, 1(07).

Castillo, L. M., Barascout, E., Arce, M., Castillo, M., y Ramírez, J. (2014). Informe Final para CAN. Proyecto de Restauración y conservación de estructuras Arquitectónicas del Monumento Nacional Guayabo (Sector Calzada Caragra (rasgo N 35), Montículos en forma de 8 (rasgos N 31-32 y N 33-34) y Plaza Mayor (rasgo N 30)). San José: Sistema Nacional de Áreas de Conservación.

Criado, F. (1999). Del terreno al espacio: planteamientos y perspectivas para la Arqueología del Paisaje. Criterios y Convenciones en Arqueología del Paisaje (CAPA). Grupo de investigación en Arqueología del Paisaje. Universidad de Santiago de Compostela. España.

Derrida, J. (1972). Limited Inc. Trad. Samuel Weber y Jeffrey Mehlman. Johns Hopkins University Press and Editions de Minuit, Estados Unidos.

Diccionario de arquitectura y construcción. http://www.parro.com.ar/index.php. Consultado 19/02/2017, 14:00 h.

Florescano, E. (1997). "El patrimonio nacional. Valores, usos, estudio y difusión", en $E I$ patrimonio nacional de México I (pp. 15-27). México: cnca/fce, .

Gándara, M. (1999). "La interpretación temática y la conservación del patrimonio cultural", en E. Cárdenas (coord.), 60 años de la enah (pp. 453-477). México: enah.

Gertz, A. (1996). El saqueo arqueológico: lento suicidio. Arqueología Mexicana, (21), pp. 22-29.

González, F. (1996). El saqueo arqueológico. La opinión de un arqueólogo. Arqueología Mexicana, (21), pp. 30-32.

Heidegger, M. (1927). Ser y Tiempo. Escuela de Filosofía Universidad ARCIS. Edición electrónica tomada de www.philosophia.cl/

Kuhn, T. (1962). La Estructura de las Revoluciones Científicas. Trad. de Agustín Contin. Fondo de Cultura Económica. México.

Mumford, L. (1979). La ciudad en la historia: Sus origenes. Ediciones Infinito, Buenos Aires.

Lakatos, I. (1978). La metodología de los programas de investigación cientifica. Editorial Alianza. Madrid.

Lyotard, J. (1994). La condición posmoderna. Madrid: Cátedra.

Litvak, J. (1979). El patrimonio arqueológico nacional. Un problema de concepto y proceso. Cuadernos de Arquitectura y Conservación del Patrimonio Artístico, (4-5), 70-74.

Orosco, G. (2002). Diccionario arquitectónico ilustrado. Centro de Conservación del Patrimonio Artístico y Arquitectónico, Bolivia.

Pelayo G. y Gross, R. (1972). Diccionario Larousse. París: Larousse.

Real Academia Española. (2014). Diccionario de la lengua española (23a . ed). Madrid: Espasa. 
Salgado, S., Hoopes, J., Arias, M., Maloof, G. y Aguilar, M. (2009). Informe final Proyecto "Contribuciones a la Arqueología de Suerre". Documento Vicerrectoría de Investigación. San José: Universidad de Costa Rica.

Salgado, S.; Hoopes, J.; Aguilar, M. y Fernández, P. (2013). El sitio Nuevo Corinto (L-72NC): una aldea cacical. Costa Rica: Universidad de Costa Rica, Universidad de Kansas y Fundación de Museo del Banco Central.

Thomas, J. 1993: "The politics of Vision and the Archaeologies of Landscape". En B. Bender (ed.): Landscape. Politics and Perspectives. Inglaterra: Berg Publishers.

Ward, B. (1972). Dictionary of building preservation. Nueva York: J. Wiley.

Wolfgang P. (1964). Encyclopedia of modern architecture. New York: H. N. Abrams.

Wittgenstein, L. (1988). Investigaciones filosóficas. Barcelona: Editorial Crítica.

Wittgenstein, L. (1921). Tractatus Logico-Philosophicus. Kegan Paul, Trench, Trubner \& CO., LTD. New York: Harcourt. 